\title{
Central Sleep Apnea - a Rare Cause for Acute Respiratory Insufficiency in Children. Case Report
}

Nicoleta Aurelia POPESCUa, Marcela Daniela IONESCU ${ }^{a}$, , Georgiana BALANa, Simina VISANa, Eliza CINTEZA ${ }^{\mathrm{b}, \mathrm{c}}$, Diana STANESCUd, lonut GOBEJ ${ }^{\mathrm{e}}$, Mihaela BALGRADEAN ${ }^{\mathrm{b}, \mathrm{f}}$

${ }^{a} 1^{\text {st }}$ Pediatric Department, "MS Curie" Emergency Children's Hospital, Bucharest, Romania b"Carol Davila" University of Medicine and Pharmacy, Bucharest, Romania

"Pediatric Cardiology Department, “M. S. Curie” Children's Emergency Hospital, Bucharest, Romania

${ }^{d}$ Radiology Department, "M. S. Curie" Children's Emergency Hospital, Bucharest, Romania

ePediatric Neurosurgery Department, "M. S. Curie” Children's Emergency Hospital, Bucharest, Romania

${ }^{\text {f}} 2^{\text {nd }}$ Pediatric Department, "M. S. Curie” Children's Emergency Hospital, Bucharest, Romania

\begin{abstract}
- ABSTRACT
Central sleep apnea is characterized by frequent cessation of breathing during sleep, resulting in repetitive episodes of insufficient ventilation and abnormalities of acid-base balance. It may be primary or secondary, and it is uncommon in children, with limited data for this population.

We present here the case of a five-year-old girl, known to have thoracolumbar myelomeningocele (for which she underwent a surgical procedure in infancy), secondary hydrocephalus (with a ventriculoperitoneal shunt) and flaccid paralysis, who was admitted in our hospital with prolonged fever syndrome, productive cough, severe dyspnea and perioral cyanosis. Following physical examination, laboratory investigations and thoracic radiography, we established the diagnosis of aspiration pneumonia with acute respiratory failure. Medical treatment with multiple systemic antibiotics, antifungal agents, systemic and inhaled bronchodilator, oxygen therapy and respiratory nursing were initiated, with favorable evolution.

During the entire hospitalization, the patient showed nocturnal respiratory rhythm disorders, with sleep apnea crisis of approximately 20 seconds and desaturation, followed by severe hypercapnic respiratory acidosis, manifestations that persisted even after the remission of pulmonary infection, raising the suspicion of an apnea syndrome. After excluding the causes of obstructive apnea, a cerebral CT scan was performed, revealing isolated fourth ventricle compressing the brainstem. The patient underwent neurosurgical intervention and postoperatively, the evolution was favorable, with remission of apnea crisis.
\end{abstract}

Keywords: central sleep apnea, isolated fourth ventricle, myelomeningocele, hypercapnia, child.

\footnotetext{
Address for correspondence:

Dr. Marcela Daniela Ionescu

"MS Curie" Emergency Children's Hospital

Tel.: 0741220099

Email:drmarcela.ionescu@gmail.com
}

Article received on the $19^{\text {th }}$ of January 2018 and accepted for publication on the $26^{\text {th }}$ of March 2018. 


\section{INTRODUCTION}

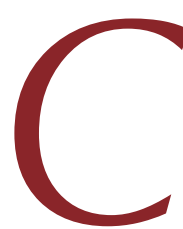

entral sleep apnea (CSA) consists of repetitive cessation or decrease of both airflow and ventilator effort during sleep, resulting in compromised gas exchange (1). It consists of an event of minimum 10 or more seconds of respiratory pause, without detectable respiratory effort, accompanied by tachycardia, cyanosis, pallor or pronounced hypotonia, and it is diagnosed when at least $50 \%$ of events are central (during polysomnography monitoring) (2). In contrast to obstructive sleep apnea, in which respiratory efforts are discernible, CSA is defined by a lack of respiratory movements during the period of airflow cessations $(1,3)$.

Central sleep apnea is uncommon in the general population, with a higher prevalence among older adults and those with associated comorbid conditions such as cardiovascular and cerebrovascular diseases. It is rare in children over one year of age and data are still limited. The causes are dominated by neurosurgical disorders (4).

Central sleep apnea can either be primary, the etiology of which is not entirely understood, or secondary. Primary CSA is characterized by repetitive absence of breathing effort and airflow lasting for 10 seconds or more (five or more central apneas per hour of sleep). These patients have a low-normal $\mathrm{PaCO}_{2}$ and do not associate hypoxemia. Secondary CSA can arise from various forms of events: Cheyne-Stokes breathing pattern, associated with an underlying medical condition, high-altitude periodic breathing, use of medications or substances $(2,5)$. There is an overlap between CSA and obstructive sleep apnea, suggesting that common pathophysiological mechanisms are likely to be involved and making it difficult to distinguish between them. Typically, CSA is considered to be the primary diagnosis when more than $50 \%$ of apneas are scored as central (cessation of breathing without any respiratory effort, lasting for 10 seconds) $(1,3)$.

Mechanisms of normal ventilation are tightly regulated by feedback loops that involve central and peripheral chemoreceptors which respond to changes in levels of arterial $\mathrm{CO}_{2}$ and $\mathrm{O}_{2}$, intrapulmonary vagal receptors, respiratory muscles and respiratory control centers in the brain stem. Any disturbances that occur in the reflex arch (chemoreceptors, respiratory control centers or respiratory muscles) may induce apnea $(1,6)$. The mechanisms involved may vary considerably but the main underlying feature is represented by unstable ventilation drive during sleep (3).

Central nervous system disease (tumor, trauma, infections, hemorrhage, malformation or genetic causes - such as congenital central hypoventilation syndrome), neuromuscular disorders (myasthenia gravis, amyotrophic lateral sclerosis, metabolic myopathies, post-polio syndrome), severe abnormalities in respiratory mechanics (kyphoscoliosis) or use of respiratory depressant drugs (opioid) may induce hypoventilation. These patients will have daytime hypercapnia during wakefulness. During sleep, the wakefulness stimulus and other behavioral influences to breath are suppressed, resulting in alveolar hypoventilation and worsening hypercapnia, severe enough to induce central apneas. Ventilation is restored during arousal from sleep $(2,3,6)$.

Central sleep apnea also results if arterial level of carbon dioxide is lowered below a sensitive threshold. This mechanism usually occurs due to hyperventilation induced by different stimuli (such as hypoxia), producing hypocapnia and consecutive central apnea. The central apnea restores eucapnia or induces hypercapnia, so that breathing is restarted. Thus, the process is cyclically repeated, alternating periods of apnea or hypopnea with hyperpnea during sleep time, so that central apneas are recurrent, not isolated events. This category includes Cheyne Stokes breathing, idiopathic central sleep apnea and altitude-induced breathing instability. Patients usually have some predisposing factors (congestive heart failure, renal failure, stroke) (1-3).

Multiple apnea episodes during sleep time result in hypoxia (evidenced by oxygen desaturations below $90 \%$ in room air). Hypoxia induces systemic cardiovascular effects: reduction of myocardium contractility, heart failure, arterial hypertension (often resistant to treatment), atrial or ventricular arrhythmias, ischemic heart disease, and pulmonary hypertension. Besides the cardiovascular complications of sleep apnea, hypoxia also induces endocrinological and metabolic abnormalities such as hyperinsulinemia and hyperglycemia (8). It is essential to detect nocturnal gas exchange abnormalities before complications occur.

Patients with CSA usually present disrupted sleep with frequent awakenings, day time sle- 
epiness, poor sleep quality, difficulty falling asleep, inattention, and fatigue. Insomnia is more prominent than in obstructive sleep apnea which is dominated by daytime drowsiness. Central sleep apnea is typically associated with other medical disorders, so that patients report symptoms of their coexisting pathology $(1,9)$. Also, there are no specific physical findings for this type of sleep apnea. Usually, physical examination is characteristic for the associated condition.

The essential step of diagnostic approach is to document abnormalities of oxygenation during sleep. Laboratory investigations are not helpful in the diagnosis of CSA, except for those attempting to identify disturbances in arterial blood gases. Underlying causes should be evaluated (including metabolic studies) (1, 2).

Imaging exam findings are also non-specific but can identify the underlying cause of CSA. However, they are not routinely used. For example, patients with central nervous system abnormalities may have specific aspects of cerebral CT or MRI examination (10).

Most diagnoses of CSA use polysomnography study, which is currently considered the "gold standard" for evaluating sleep-disordered breathing. It is a complex measurement that typically includes electroencephalogram, electrooculogram and electromyogram leads, a nasal/ oral thermistor to assess airflow, thoracic and abdominal monitors for monitoring excursion during breathing, electrocardiogram and leg leads, a snoring microphone and pulse oximetry (11). Oxygen desaturation may also be screened using overnight pulse oximetry alone, although it may underestimate the degree of sleep apnea (12).

The pathophysiologic factors contributing to the CSA varies, so that treatment approach varies considerably. Identifying and eliminating the underlying medical condition are essential (reduction of opioid medication, weight loss, improving cardiac status, neurosurgery) (3). Several different treatments used in CSA include $\mathrm{O}_{2}$ therapy (used in non-hypercapnic CSA patients with high chemosensitivity, but with the concern that may have cardio-depressant effects), inhaled $\mathrm{CO}_{2}$ (requiring larger studies), non-invasive ventilation (continuous positive airway pressure, bilevel positive airway pressure), adaptive servo-ventilation (very efficient for treatment of CSA, especially Cheyne-Stokes breathing) (2, 3, $13,14)$. Although several studies have shown the potential for different treatment options, larger randomized trials are required to determine the long-term efficacy and safety of therapeutic options in CSA, especially in children, for whom data are still little known.

\section{CASE REPORT}

A girl aged five years and six months was transferred to our clinic from a territorial hospital with a history of prolonged fever syndrome, frequent productive cough, followed by marked expiratory dyspnea and perioral cyanosis, lasting for three weeks prior admission. She had significant past medical history of global development delay, thoracolumbar myelomeningocele (for which she underwent a surgery procedure at the age of two months), secondary hydrocephalus (with ventriculoperitoneal shunt inserted), flaccid paraplegia, neurogenic bladder with secondary vesicoureteral reflux - IVth grade.

Physical examination at presentation revealed an underweight pale child, with peripheral edema, fever $\left(39^{\circ} \mathrm{C}\right)$ and mediocre general state, in severe respiratory distress, with tachypnea (40 rpm), retractions, oxygen desaturation of up to $60 \%$ under oxygen therapy, predominantly during nocturnal sleep. Pulmonary auscultation detected symmetric breath sounds, multiple crackles, disseminated rhonchi. She was tachycardic (HR $160 \mathrm{bpm}$ ), with high blood pressure $(140 / 110 \mathrm{mmHg}$ ) and needed intermittent urinary catheterization. Because of difficulties in feeding due to swallowing disorder, a nasogastric tube was inserted. There was moderate generalized hypotonia, with flaccid paraplegia, so that the patient could not walk or sit without support. She was unable to speak and presented agitation alternating with somnolence.

Laboratory investigations showed leukocytosis (WBC 32.800/mmc) with neutrophilia (59\%), elevated inflammatory tests (CRP $40 \mathrm{mg} / \mathrm{L}$, procalcitonin $>2 \mathrm{ng} / \mathrm{mL}$ ), moderate normocytic normochromic anemia $(\mathrm{Hb} 9 \mathrm{~g} / \mathrm{dL}, \mathrm{MCV} 82 \mathrm{fL}$, $\mathrm{MCH} 27$ pg). Other blood tests were normal (including sterile urine and blood cultures), except for acid-base balance, with a tendency towards respiratory acidosis and hypercapnia. Lumbar puncture could not be performed because of the history of thoracolumbar myelomeningocele.

Thoracic radiography revealed accentuated interstitial drawing in both pulmonary fields and 
the ventriculoperitoneal shunt passing laterally to the right of the spine. An abdominal ultrasound was performed to evaluate the ventriculoperitoneal shunt. A small amount of fluid between intestinal loops was found and IVth grade ureterohydronephrosis was described.

The diagnosis of aspiration pneumonia with acute respiratory insufficiency was formulated.

Medical treatment with multiple systemic antibiotics (Ceftazidime, Teicoplanin, Piperacillin/ Tazobactam), antifungal agents, systemic and inhaled bronchodilator, associated with enteral nutrition, oxygen therapy and respiratory nursing were initiated.

The patient evolution was favorable, with fever remission, normalization of pulmonary auscultation, reduced cough frequency, normalization of blood count and inflammatory tests, normalization of chest X-ray. However, despite clinical and laboratory improvement, during the entire hospitalization, the patient presented nocturnal respiratory rhythm disorders, with sleep apnea crises of approximately 20 seconds and desaturation of up to $60 \%$ under oxygen supplementation through nasal cannula, followed by morning decompensated hypercapnic respiratory acidosis $\left(\mathrm{pH} 7.23 ; \mathrm{PaCO}_{2} 82 \mathrm{mmHg}\right.$; $\mathrm{HCO}_{3} 23 \mathrm{mmol} / \mathrm{L}$ ). These events caused frequent fragmentation of sleep and, consecutive, drowsiness alternating with periods of daytime agitation.

A sleep apnea syndrome was suspected. The ENT consultation excluded the presence of obstructive peripheral apnea. Oral examination revealed an arched palate, with no maxillary retrusion or micrognathia, normal tongue and Mallampati class II. Thus the diagnostic of central apnea was taken into consideration. Echocardiography was normal and 24 hour Holter monitoring excluded arrhythmia and atrioventricular conduction abnormalities.

A polysomnography study could not be performed at that moment, but the patient was continuously monitored. The apnea crisis was characterized by minimum 20 seconds of respiratory pause, without detectable respiratory effort, accompanied by cyanosis, pallor and severe oxygen desaturation. Ventilation was only possible by awakening from sleep.

A cerebral CT scan was performed and revealed isolated closed fourth ventricle, compressing the brainstem and the cerebellum (Fi- gure 1). The diagnosis of central sleep apnea secondary to isolated fourth ventricle compressing the brainstem was established. The pediatric neurosurgery department was consulted for the possibility of surgical treatment. The patient underwent a surgical intervention consisting of communication between the cystic fourth ventricle and the latero-bulbar systems, using infraoccipital approach. This procedure determined the decompression of cerebral trunk and especially of the bulb, leading to complete remission

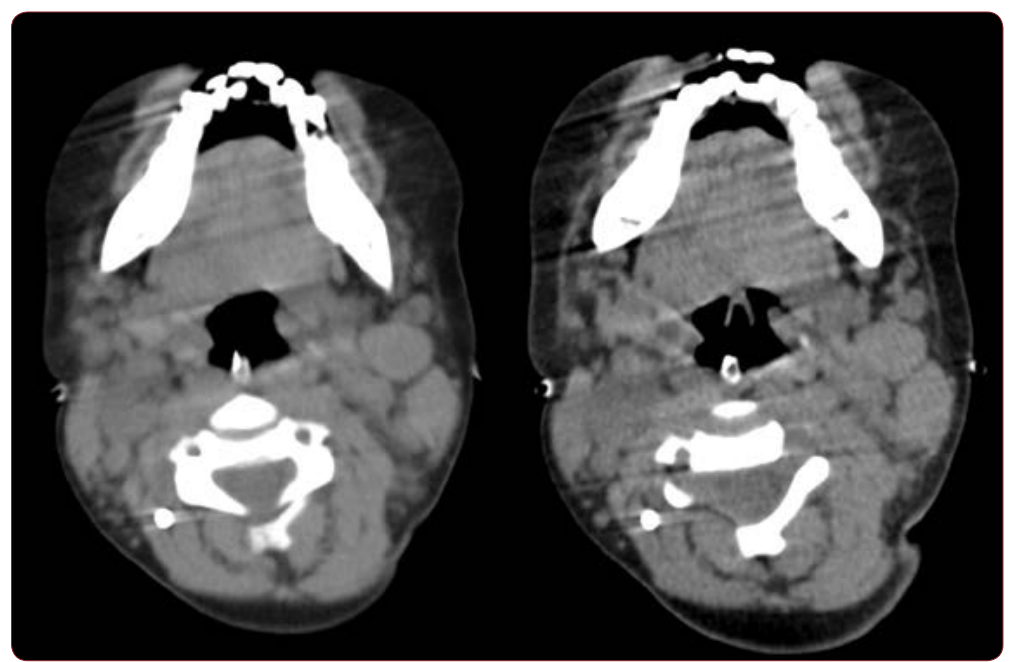

FIG URE 1. Preoperative cerebral CT scan. Isolated closed fourth ventricle, compressing the brainstem and the cerebellum

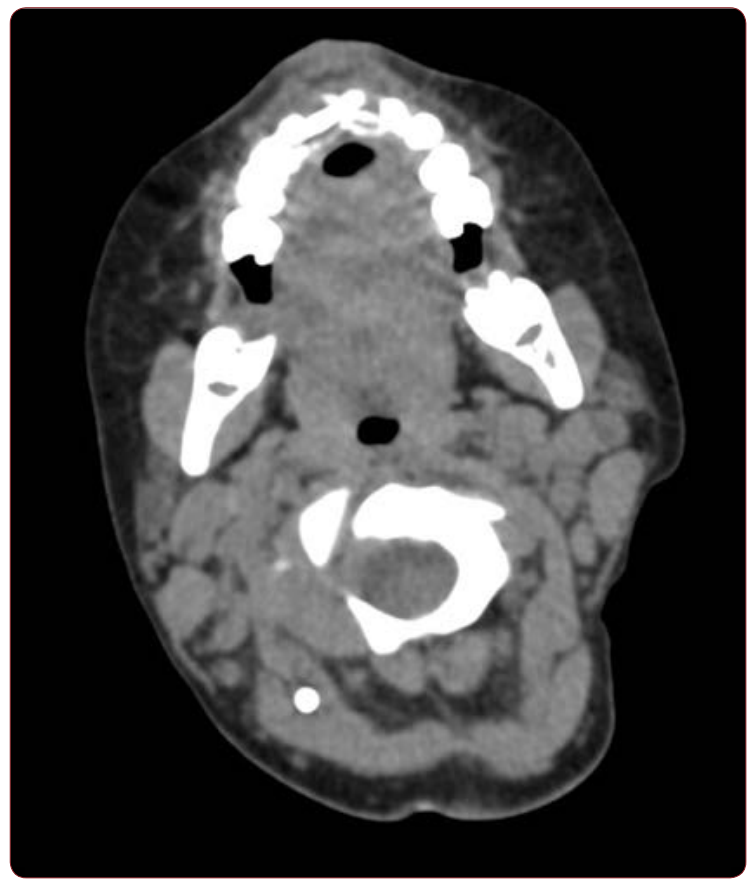

FIG URE 2. Postoperative cerebral CT scan.

Decompression of brain stem, and especially of the medulla 
of the respiratory symptomatology manifested as central apnea (Figure 2).

\section{DISCUSSION}

C entral sleep apnea is a rare condition in children, the causes being dominated by neurosurgical abnormalities.

Patients with myelomeningocele are known to have sleep apnea depending on functional level of the spinal lesions, brainstem abnormalities (congenital/acquired), disorders of upper airway maintenance, and sleep state (15).

We were faced to an unusual mechanism of CSA in a patient with multiple comorbid states. That was the isolated fourth ventricle, a very rare complication of the thoracolumbar myelomeningocele with drained secondary hydrocephalus (using ventriculoperitoneal shunt). The closure of the IV ventricle caused compression on the brainstem and subsequently central apnea phenomena. Neurosurgical resolution resulted in definitive remission of respiratory symptomatology.

Isolated or encysted fourth ventricle is a rare late complication following insertion of lateral ventricular shunt for hydrocephalus. Caudal and rostral obstruction of the fourth ventricle isolates it from the rest of ventricular system. The choroid plexus continues to produce cerebrospinal fluid, leading to subsequent dilation of the fourth ventricle and progressive compression of adjacent structures (brainstem, cerebellum) (16).

In our case, CSA was the unveiling phenomenon of isolated fourth ventricle. The diagnosis was initially concealed by respiratory failure secondary to aspiration pneumonia. The persistence of frequent exclusive sleep apnea crisis after remission of acute respiratory infection and marked hypercapnia (predominantly nocturnal and matinal) has raised suspicion of an apnea syndrome. Nocturnal pulse oximetry, showing desaturation of up to $60 \%$, and arterial blood gases monitoring, revealing severe respiratory hypercapnic acidosis, sustained the diagnosis. Following the ENT consultation, the obstructive causes of apnea were excluded. Cardiovascular disorders were also excluded.

Being in front of a patient with myelomeningocele and ventriculoperitoneal shunt, central apnea was taken into consideration and a cerebral CT scan was performed to exclude different brainstem abnormalities. Isolated fourth ventricle was revealed and postoperative evolution was favorable, with definitive remission of CSA. Thus, identification of the cause was of utmost importance for improving the clinical condition of our patient.

Conflicts of interest: none declared. Financial support: none declared.

\section{R}

1. Malhotra A, Owens RL. What Is Central Sleep Apnea? Respir Care 2010;9:1168-1178.

2. Muza RT. Central sleep apnoea - a clinical review. J Thorac Dis 2015;5:930-937.

3. Eckert DJ, Jordan Amy S, Merchia P, Malhotra A. Central Sleep Apnea Pathophysiology and Treatment. Chest 2007;2:595-607.

4. Felix O, Amaddeo A, Olmo Arroyo, Zerah M, Puget S, et al. Central sleep apnea in children: experience at a single center. Sleep Med 2016;25:24-28.

5. American Academy of Sleep Medicine. International Classification of Sleep Disorders, $3^{\text {rd }}$ ed, American Academy of Sleep Medicine, Darien, IL 2014.

6. Khoo MC. Determinants of ventilatory instability and variability.
Respir Physiol 2000;2-3:167-182.

7. Bradley TD, Philipson EA. Central sleep apnea. Clin Chest Med 1992;13:493-505.

8. Dybala A, Dyczko M, Makaruk B, Kicinski P, Bartoszek E, Myslinski W. Central sleep apnea - a case report. Curr Issues Pharm Med Sci 2014;1:14-16.

9. Eckert DJ, Jordan AS, Merchia P, Malhotra A. Central sleep apnea: Pathophysiology and treatment. Chest 2007;2:595-607.

10. Woughter M, Perkins AM, Baldassari CM. Is MRI Necessary in the Evaluation of Pediatric Central Sleep Apnea? Otolaryngol. Head Neck Surg 2015;6:1031-1035.

11. Bloch KE. Polysomnography: a systematic review, Technol. Health Care 1997;4:285-305.
12. Douglas NJ, Thomas S, Jan MA. Clinical value of polysomnography. Lancet 1992;8789:347.

13. Franklin KA, Eriksson $P$, Sahlin $C$, et al. Reversal of central sleep apnea with oxygen. Chest 1997;111:163-169.

14. Hoffstein V., Slutsky A.S. Central sleep apnea reversed by continuous positive airway pressure. Am Rev Respir Dis 1987;135:1210-1212.

15. Waters KA, Forbes $P$, Morielli A, Hum $C$, et al. Sleep-disordered breathing in children with myelomeningocele. J Pediatr 1998;4:672-681.

16. Khalid A. Nannapaneni R, Hamandi K. Case Report The isolated fourth ventricle. BMJ Case Rep 2013; Published online 2013 Apr 3. 